\title{
Facilitating Practice Transformation in Frontline Health Care
}

\author{
Robert L. Pbillips, Jr, MD, MSPH ${ }^{1}$ \\ Deborab J. Coben, PbD ${ }^{2}$ \\ Artbur Kaufman, $M D^{3}$ \\ W. Perry Dickinson, $M D^{4}$ \\ Samuel Cykert, $M D^{5,6}$ \\ ${ }^{1}$ Center for Professionalism \& Value in \\ Health Care, Lexington, Kentucky \\ ${ }^{2}$ Department of Family Medicine, Oregon \\ Health \& Science University, Portland, \\ Oregon
}

${ }^{3}$ Office for Community Health, University of New Mexico, Albuquerque, New Mexico

${ }^{4}$ Department of Family Medicine, University of Colorado School of Medicine, Aurora, Colorado

${ }^{5}$ Division of General Medicine and Clinical Epidemiology, School of Medicine, University of North Carolina, Chapel Hill, North Carolina

${ }^{6}$ Cecil G. Sheps Center for Health Services Research, University of North Carolina, Chapel Hill, North Carolina

Conflicts of interest: guest editors report none; furthermore, guest editors were not involved in the peer review or acceptance decisions about articles on which they are authors.

\section{CORRESPONDING AUTHOR}

Robert L. Phillips, Jr, MD, MSPH Center for Professionalism \& Value in Health Care

1648 McGrathiana Pkwy, Ste 550

Lexington, KY 40511

bphillips@theabfm.org
Ann Fam Med 2019;17:S2-S5. https://doi.org/10.1370/afm.2439.

$\mathrm{T}$ This supplement to the Annals of Family Medicine brings together early learning from multiple examples of health extension and practice transformation support, with the goal of informing future efforts to improve our health care system. As this supplement is published, 2 major federal investments testing health extension, a model of practice facilitation, to achieve practice transformation will be ending. One is the $\$ 112$ million, multiregion EvidenceNOW: Advancing Heart Health in Primary Care initiative of the Agency for Healthcare Research and Quality (AHRQ), a multistate research effort to test the health extension model., ${ }^{1,2}$ The other is the nearly $\$ 700$ million Transforming Clinical Practice Initiative (TCPI) of the Centers for Medicare \& Medicaid Services (CMS), a practice facilitation demonstration project touching all 50 states that aimed to support 140,000 clinicians. $^{3}$ The response to the call for papers for this supplement garnered 50 submissions. Although we are not able to publish all of them, work not published here will also contribute to what has been learned from these large federal investments.

The history of how we got to EvidenceNOW and TCPI is important, and the special report by Kaufman et $\mathrm{al}^{4}$ in this supplement reminds us of the history behind Section 5405 of the Patient Protection and Affordable Care Act (ACA) that authorized the Primary Care Extension Program. ${ }^{5}$ This program was modeled after the Cooperative Extension Program (US Department of Agriculture), which revolutionized farming in the United States over the last 100 years by testing and speeding dissemination of innovation. Its application to health care was pilot-tested by the Regional Extension Centers under the Health Information Technology for Economic and Clinical Health Act of 2009. The ACA authorized but did not fund the Primary Care Extension Program ${ }_{i}$ however, AHRQ used this authorization to launch a pilot project in 2011, Infrastructure for Maintaining Primary Care Transformation (IMPaCT), with 4 states, which Kaufman et $\mathrm{a}^{4}$ describe. The success of this pilot project led to EvidenceNOW and further supported the decision by CMS to invest in the TCPI demonstration project. Although authorization of the Primary Care Extension Program remains law, it is unclear how these important demonstration projects will translate into new programs that can continue to speed development and dissemination of innovation in health care. Cooperative Extension has demonstrated considerable returns on investment, both in the United States and abroad. ${ }^{5}$ Without sustained investment in a health care extension, however, it is difficult to achieve a similar return on investment.

Facilitation can sometime help practices figure out how to return to their roots by focusing on core functions of primary care. Continuity is a basic, high-value function of primary care shown to be related to improved patient outcomes, reduced costs, and greater satisfaction of both patients and clinicians. Yet, it is not routinely measured or supported in clinical practice. Gukasyan and Wong ${ }^{6}$ describe their development and 
implementation of an empanelment toolkit in safetynet clinics that were part of the Los Angeles Practice Transformation Network. The authors' expressed goal was to support continuity, and the effort required considerable practice facilitation for implementation and routine use. Practice facilitation is necessary not only for the novel, but often for the fundamental functions.

Facilitation is sometimes necessary when introducing new tools that enable small practices to expand their capacities. Pariser and her team at the University of Toronto ${ }^{7}$ extended a decade-old model of interdisciplinary care into a virtual, telemedicine consultation environment to support practices that did not have interdisciplinary teams and were struggling to adequately care for very complex patients. Hour-long consultations produced a robust set of patient-informed recommendations that reflected the interplay between polypharmacy, functional disability, social determinants of health, and chronic physical and mental health conditions. These consultations reduced emergency department visits and hospitalizations, and were generally agreeable to both physicians and patients. Practices that cannot bring expanded patient services into their offices need help when introducing virtual tools that produce the same functionalities, but that might be too complex otherwise.

Wagner and colleagues at the University of Alberta ${ }^{8}$ illuminate differences in primary care team mental models and implications for supporting transformation in practices that are not early adopters. They found a range of practice models from "the doctor takes care of patients and hires some people to help her/him" to "we take care of patients," with varying levels of care delegation in between. Early adopters tend to function in team-based models, spreading authority among team members to help in decision making, are more willing (and likely) to experiment with innovations, and can hold big-picture ideas about how innovations fit their practice vision. The authors suggest that moving beyond the early adopter phase will require even more coaching, skill building, and team development. Understanding these differences between early adopters and other practices not only is critical for facilitators to support practice transformation, but also identifies culture change as having a potentially important role.

Understanding which practice facilitation modalities improve care remains an open question. Parchman and his team ${ }^{9}$ conducted a randomized controlled trial in smaller practices across 3 states, offering the practices various types of implementation support plus facilitation. They found that large proportions of practices did not engage in shared learning opportunities or educational outreach visits, both of which provided practices the opportunity for connecting with colleagues at other practices. This lack of engagement might be a sign of workload in busy practices. The study further found that all practices improved, regardless of the combination of implementation strategies offered. Their results suggest that effective facilitation may need to work at relationship and engagement in order to compete with existing practice priorities and stressors. This is not to say that remote practice facilitation will not work at all or for at least some changes, however. Adler and his TCPI team ${ }^{10}$ developed remote practice coaching for nearly 3,000 optometrists in all 50 states to promote provision of urgent eye care to reduce emergency department use. Practice facilitators (here called quality improvement advisors) remotely conducted practice assessments, established goals with each practice, and assisted with implementing iterative plan-do-study-act cycles. Electronic reporting demonstrated increases in urgent eye care visits with associated cost reductions of more than $\$ 150$ million over 13 months.

It is important to remember that practice transformation can induce stress, too, as Grumbach and his team ${ }^{11}$ found. Their study further showed that team members may not experience this stress in the same direction. They assessed burnout among clinicians and staff engaged in primary care redesign over 7 years and found variation in burnout, particularly that staff may experience rising burnout when clinicians' is in decline, and they surmise that "primary care transformation requires continuing efforts to promote meaningful work and sustainable workloads among all members of the primary care team." This may be particularly true when work is simply being shifted. If, instead, practices' resources are increased while work is redistributed, all involved may benefit. This strategy was used in the Primary Care Redesign team-based model tested by Smith and colleagues. ${ }^{12}$ This model increased the ratio of medical assistants to clinicians from about 1:2 to 2.5:1 while also expanding the role of medical assistants, and was facilitated by practice coaches. Clinician burnout was reduced by one-half with simultaneous improvements in quality, patient access, and clinician panel size-all while maintaining staffing costs. Most practice transformation efforts do not increase resources, and this study demonstrates that doing so can improve all aspects of the quadruple aim, ${ }^{13}$ including at least holding per-visit costs constant. Although clinician burnout was reduced significantly, as in the study by Grumbach and colleagues, ${ }_{11}^{11}$ staff stress scores increased initially before returning to baseline.

Sometimes, practice facilitation seems straightforward as in the case of Guck and his colleagues, ${ }_{1}^{14}$ who describe a single-practice innovation as an approach to high-risk patients. They implemented an interprofessional collaborative practice (IPCP) model within an 
academic practice that included staff and clinician training, patient care preparation, and care-planning conferences. Analysis of patient outcome data collected for a year before and a year after implementation of the IPCP model found meaningful improvements on multiple outcome measures. It is unlikely that this model would be easily spread without practice facilitation.

A qualitative study of small, independent practices by Rogers et $\mathrm{a}^{15}$ is instructive about the complexity of support offered by practice facilitators. Specific supports that were highlighted included connecting practices to the external health care environment, often through teaching and information sharing, and providing electronic health record (EHR) and data expertise, commonly by teaching functionality and providing technical assistance. These small practices noted 3 key benefits of practice facilitation: (1) creating awareness of quality gaps, (2) connecting practices to information, resources, and strategies, and (3) optimizing the EHR for quality improvement goals. Consistent with these findings, Khanna and colleagues in the Garden Practice Transformation Network ${ }^{16}$ focused on translating quality and cost data into a practice transformation analytics dashboards for practices. Their study echoes both a qualitative study of family physicians participating in TCPI about their reasons for doing so, ${ }^{17}$ and a study by the EvidenceNOW evaluation team, ESCALATES.$^{18}$ The dashboard became a tool for practice facilitators to help practices develop quality improvement plans and celebrate success, but fewer than one-half of practices still found the information to be actionable. Khanna et $\mathrm{al}^{16}$ reiterate the lesson that "smaller practices are most likely to lack resources to review, interpret, and act on data." Gritzer and colleagues in the Garden Practice Transformation Network ${ }^{19}$ describe an innovation that entailed facilitating small practices' use of patient portals that offers several exemplars of the study by Rogers et al ${ }_{1}^{15}$ namely, practice facilitator interventions with EHR vendors to enable patient portal functionality, help practices understand related return on investment, teach them to use portal functions, and assist them in realizing the role of patients in informing and improving quality. These studies are important for showing that effective practice facilitation is not just technical assistance, but rather may need to also include help with using quality data and building confidence and skills, sharing learning, and even conducting advocacy.

Addressing social determinants of health is increasingly discussed as a necessary function of outpatient care. Community-oriented primary care is a well-tested and systematic way for practices to define practice community, then assess and address community needs, evaluate outcomes, and repeat..$^{20}$ This approach has proven difficult to implement for several reasons, including problems with defining "community." Rock and colleagues ${ }^{21}$ tested the relationship between actual and clinician-predicted geographic service area and found vast discrepancy. They conclude that "practices need tools to better understand the communities they serve before they can be expected to undertake population-level interventions."

What comes after EvidenceNOW and TCPI is an open question. How the information learned will inform future efforts to support practice transformation is, too. Letourneau et al ${ }^{22}$ outline a multistate effort by the Network for Regional Health Improvement and its 30-member regional health improvement collaboratives to increase access to opioid use disorder treatment in several states. They discuss a general approach to practice facilitation able to adapt to variation in state resources and policies, and in local relationships. The authors describe regional health improvement collaboratives as independent, nonprofit organizations composed of multiple stakeholders who come together to improve health and health care, and note that they must include health care professionals, payers (health plans), health care purchasers (employers), and consumers. Collectively, these collaboratives cover 32 states and already serve as TCPI entities, quality improvement organizations, regional extension centers, and health information exchanges. Opioid use disorder is an important epidemic for which practice facilitation could increase the rate of best-practice adoption, but its collaborative management also presents an exemplar of the benefits of practice facilitation in addressing urgent health issues to come.

Several of the articles in this supplement are the early products of nearly $\$ 800$ million invested by federal health agencies to test transformation facilitation in thousands of practices across the United States. Collectively, they tell a story of practices needing relationships and real support in achieving meaningful improvement, if not fundamental transformation. These articles describe strategies for helping practices use technology to strengthen relationships with patients and to offer complex patients expanded services. They capture the complexity and spectrum of practice culture and the need to meet them where they are in order to help the difficult process of change. Most speak to the need for meaningful relationships and work, without which in-person support does not work, and some show how remote support can work when it is available. Two articles ${ }^{11,12}$ remind us that the hard work of change can produce stress and even burnout, sometimes differently across the team, but others suggest that engaging the whole team can enable meaningful improvements. Many are instructive 
about the important functions of practice facilitators and how these functions may differ by practice type, offering guidance on preparing this workforce.

Most practices lack time, energy, and resources to make these changes on their own, and most lack means of learning about the policies pushing them to change or examples from which they can learn. Farming was in a similar situation a century ago, prompting federal and state governments set up the Cooperative Extension system that is still facilitating and speeding transformation of the process whereby that nation's nutritional needs are met. It is one of our most emulated programs around the world, and evidence of its investment returns make it one of the most supported federal programs. Practice facilitation, health extension, and other forms of support for practice transformation and community health improvement are important systems-level interventions to improve health care and accomplish the quadruple aim. ${ }^{13}$ The benefits of such improvements generally accrue across multiple stakeholders, making funding of such "public good" efforts a challenge. Further results from EvidenceNOW, TCPI, and other large-scale practice and community health improvement efforts will emerge over the next few years and should inform whether and how the United States will support practice transformation facilitation and community health improvement efforts in the future.

To read or post commentaries in response to this article, see it online at http://www.AnnFamMed.org/content/17/Suppl_1/S2.

Key words: practice transformation; organizational change; innovation; quality improvement; professional practice; health extension; outreach; practice facilitation; primary care; burnout; health information technology; practice-based research

Submitted June 13, 2019; submitted, revised, June 18, 2019; accepted July 1, 2019.

Funding support: This supplement was supported by Funding Opportunity Number CMS-1L1-15-002 from the U.S. Department of Health $\varepsilon$ Human Services (HHS), Centers for Medicare \& Medicaid Services.

Disclaimer: The contents provided are solely the responsibility of the authors and do not necessarily represent the official views of HHS or any of its agencies.

Acknowledgments: The authors wish to thank Dr Kevin Grumbach and Dr Jim Mold who contributed greatly as part of the guest editorial team for this Annals of Family Medicine supplement.

\section{References}

1. Meyers D, Miller T, Genevro J, et al. EvidenceNOW: Balancing primary care implementation and implementation research. Ann Fam Med. 2018;16(Suppl 1):S5-S11.

2. Cohen DJ, Balasubramanian BA, Gordon L, et al. A national evaluation of a dissemination and implementation initiative to enhance primary care practice capacity and improve cardiovascular disease care: the ESCALATES study protocol. Implement Sci. 2016;11(1):86.
3. Centers for Medicare \& Medicaid Services. Fact sheet: Transforming Clinical Practice Initiative Awards. https://www.cms.gov/newsroom/ fact-sheets/transforming-clinical-practice-initiative-awards. Created Sep 29, 2015. Accessed Jul 28, 2019.

4. Kaufman A, Dickinson WP, Fagnan LJ, Duffy FD, Parchman ML, Rhyne RL. The role of health extension in practice transformation and community health improvement: lessons from 5 case studies. Ann Fam Med. 2019;17(Suppl 1):S67-S72.

5. Phillips RL Jr, Kaufman A, Mold JW, et al. The Primary Care Extension Program: a catalyst for change. Ann Fam Med. 2013;11(2): 173-178.

6. Gukasyan S, Wong M. An empanelment toolkit for the safety-net clinic setting. Ann Fam Med. 2019;17(Suppl 1):S84.

7. Pariser P, Pham T-N(T), Brown JB, Stewart M, Charles J. Connecting people with multimorbidity to interprofessional teams using telemedicine. Ann Fam Med. 2019;17(Suppl 1):S57-S62.

8. Wagner KK, Austin J, Toon L, Barber T, Green LA. Differences in team mental models associated with medical home transformation success. Ann Fam Med. 2019(Suppl 1);17:S50-S56.

9. Parchman $\mathrm{ML}$, Anderson ML, Dorr DA, et al. A randomized trial of external practice support to improve cardiovascular risk factors in primary care. Ann Fam Med. 2019;17(Suppl 1):S40-S49.

10. Adler RN, Ferguson WJ, Antar H, et al. Transformation support provided remotely to a national cohort of optometry practices. Ann Fam Med. 2019;17(Suppl 1):S33-S39.

11. Grumbach K, Knox M, Huang B, Hammer H, Kivlahan C, WillardGrace R. A longitudinal study of trends in burnout during primary care transformation. Ann Fam Med. 2019;17(Suppl 1):S9-S16.

12. Smith PC, Lyon C, English AF, Conry C. Practice transformation under the University of Colorado's primary care redesign model. Ann Fam Med. 2019;17(Suppl 1):S24-S32.

13. Bodenheimer T, Sinsky C. From triple to quadruple aim: care of the patient requires care of the provider. Ann Fam Med. 2014;12(6): 573-576.

14. Guck TP, Potthoff MR, Walters RW, Doll J, Greene MA, DeFreece T. Improved outcomes associated with interprofessional collaborative practice. Ann Fam Med. 2019;17(Suppl 1):S82.

15. Rogers ES, Cuthel AM, Berry CA, Kaplan SA, Shelley DR. Clinician perspectives on the benefits of practice facilitation for small primary care practices. Ann Fam Med. 2019;17(Suppl 1):S17-S23.

16. Khanna N, Gritzer L, Klyushnenkova E, et al. Practice transformation analytics dashboard for clinician engagement. Ann Fam Med. 2019;17(Suppl 1):S73-S76.

17. Hansen ER, Eden AR, Peterson LE, Bishop EM, Phillips RL Jr. Experience of family physicians in practice transformation networks. J Ambul Care Manage. 2019;42(2):92-104.

18. Ono SS, Crabtree BF, Hemler JR, et al. Taking innovation to scale in primary care practices: the functions of health care extension. Health Aff (Millwood). 2018;37(2):222-230.

19. Gritzer L, Davenport M, Dark M, Khanna N. Coaching small primary care practices to use patient portals. Ann Fam Med. 2019; 17(Suppl 1):\$83.

20. Institute of Medicine Division of Health Care Services. In: Community Oriented Primary Care: A Practical Assessment: Volume I: The Committee Report. Washington, DC: National Academies Press; 1984.

21. Rock RM, Liaw WR, Krist AH, et al. Clinicians' overestimation of their geographic service area. Ann Fam Med. 2019;17(Suppl 1): S63-S66.

22. Letourneau LM, Ritzo J, Shonk R, Eichler M, Sy S. Supporting physicians and practice teams in efforts to address the opioid epidemic. Ann Fam Med. 2019;17(Suppl 1):S77-S81. 